\title{
Agitation of the syringe and release of silicone oil
}

\author{
Celso de Souza Dias Júnior (iD ${ }^{1} \cdot$ Alexandre Lima Cardoso $^{1} \cdot$ Ana Galrão de Almeida Figueiredo ${ }^{2} \cdot$ Shoko Ota $^{3}$. \\ Gustavo Barreto Melo $\mathbb{1}^{1,4}$
}

Received: 26 July 2019 / Revised: 3 December 2019 / Accepted: 10 December 2019 / Published online: 3 March 2020

(c) The Author(s), under exclusive licence to The Royal College of Ophthalmologists 2020

\begin{abstract}
Background/objectives To investigate whether agitation promotes the release of silicone oil by different models of syringe used for intravitreal injection.

Methods This lab study analyzed eight syringe models by light microscopy for the release of silicone oil under agitation (flick), without agitation, and positive controls. Fourier-Transform Infrared Spectroscopy (FTIR) was performed to identify the molecular compounds inside the syringes.

Results A total of 240 syringes were analyzed. The presence of silicone oil droplets was observed in all positive controls. When agitated by flicking, $100 \%$ of the samples of the syringes disclosed silicone oil, except the BD Plastipak syringe, which presented $40 \%$ of positivity. Without agitation, a smaller percentage of samples with silicone oil was observed. Agitation by flicking had a 265 -fold greater chance of presenting oil droplets when compared with the syringes without agitation. There was a statistically significant difference between the three conditions $(P<0.05)$. Analysis of the tip of the plunger rubber by FTIR indicated the presence of polysiloxane (silicone oil) in all models of syringe.

Conclusions Agitation of the syringe promotes the release of silicone oil. It is recommended to improve the technique of injection and the manufacture of specific syringes for ophthalmological use.
\end{abstract}

\section{Introduction}

The number of intravitreal injections has increased significantly, outnumbering cataract surgery as the most common intraocular procedure. [1] There are wellestablished indications for intraocular antiangiogenic use: AMD, diabetic macular edema, retinal vein occlusions, myopic choroidal neovascularization, and proliferative diabetic retinopathy [2].

Virtually all brands of syringes used for intraocular injection have silicone oil (SO) on their inner walls, which is added during their manufacturing, a process known as

Gustavo Barreto Melo

gustavobmelo@yahoo.com.br

1 Hospital de Olhos de Sergipe, Aracaju-SE, Brazil

2 Tiradentes University - UNIT, Aracaju-SE, Brazil

3 Chemical Analysis Laboratory, Center for Chemistry and Manufactured Goods, Institute for Technological Research, São Paulo-SP, Brazil

4 Federal University of São Paulo, São Paulo-SP, Brazil "siliconization". Among other purposes, it facilitates gliding of the plunger during injection.

Since most, if not all, syringes have not been developed specifically for intraocular use, insulin/tuberculin syringes are commonly used. The SO present in their interior can be released into the vitreous as droplets during intravitreal injection [3-7]. It might lead to an annoying complaint of floaters that, in some cases, requires vitrectomy. Although vitrectomy is considered a safe procedure, there are inevitable complications possibly associated with any surgery. The American Society of Retina Specialist 2018 Preferences and Trends Membership Survey showed that the $60.4 \%$ of US respondents have seen SO in the vitreous, while $5.2 \%$ already have performed vitrectomy to remove the oil. Moreover, $1.8 \%$ reported that patients have sought legal action because of the presence of the floaters [8].

Another important issue concerning the SO released by the syringes refers to a possible association to inflammation. A case-control study from our group showed a chronological link between noninfectious inflammatory reaction after intravitreal injection of aflibercept in six patients and the use of a specific brand of syringe subsequently shown to release a significant amount of SO [3, 9]. 


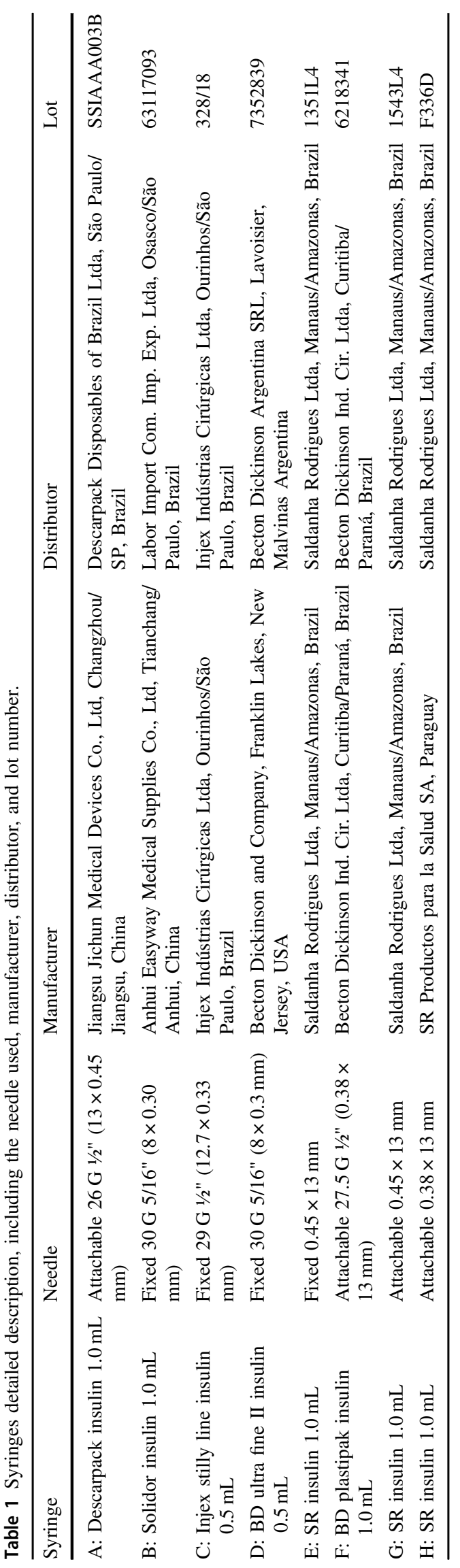

All these findings and the relevance of this topic led us to study whether agitation promotes the release of SO by different brands of syringes.

\section{Materials and methods}

Eight syringe models were analyzed for the release of SO. Detailed information about each one is presented in Table 1. All syringes were tested with their own needles. Solidor (B), Injex (C), BD Ultra fine II (D), and SR (E) have fixed needles, while Descarpack (A), BD Plastipak $(\mathrm{F})$, SR $(\mathrm{G})$, and SR $(\mathrm{H})$ have attachable needles.

Thirty syringes of each model were tested under three different handling conditions (ten syringes of each model for each condition): positive control (fluid with deliberate addition of SO) with agitation by flicking (group 1); fluid with agitation by flicking (group 2) and fluid without agitation (group 3). The fluid used for all syringes was distilled water (Samtec Biotecnologia Ltda, São Paulo, Brazil) and the SO used for the positive control was the same used in vitrectomy surgeries (RS-Oil ECS, Alchimia, Ponte San Nicolo, Italy, batch: P180020016, Ref.: RSO 010-00).

\section{Preparation of syringes}

In order to simulate the clinical conditions of intravitreal injection, $0.06 \mathrm{~mL}$ of distilled water was aspirated into all the syringes of the three groups (filled by drawing with their own needle). Only the syringes of the positive control group had addition of a drop of SO to the tip of the plunger rubber (RS-Oil ECS, Alchimia, Ponte San Nicolo, Italy, lot: P180020016, Ref.: RSO 010-00). In groups one and two, after aspiration of distilled water, five flicks were given with the syringe needle facing upward and, then, ten more flicks with the needle of the syringe facing downward. No agitation was performed in Group 3 after aspiration of the liquid. Immediately after this procedure (carried out in one syringe at a time), the fluid was ejected onto a glass slide (Yancheng Huida Medical Instruments Co., Jiangsu, China) and analyzed with a light microscope (Nikon Eclipse 200, Shanghai, China). A single investigator (CSDJr) performed all steps in all syringes in order to avoid inter-examiner variability.

\section{Microscopic analysis of syringe fluid}

For each syringe, a different slide was used and was observed using an optical microscope under $\times 100$ optical magnification. An additional 50\% digital magnification and photographic recording were used with an iPhone $6 \mathrm{~S}$ (Apple Inc., California, USA) attached to a microscope adapter (Eye2Mobile LLC, California, USA). An image 
Table 2 Qualitative data for each condition and type of syringe.

\begin{tabular}{lllcr}
\hline Syringe $N(\%)$ & Positive control & Agitation & Without agitation & $P$ value $^{\dagger}$ \\
\hline A & $10 / 10(100.0 \%)$ & $10 / 10(100.0 \%)$ & $2 / 10(20.0 \%)$ & $<\mathbf{0 . 0 0 1}$ \\
B & $10 / 10(100.0 \%)$ & $10 / 10(100.0 \%)$ & $10 / 10(100.0 \%)$ & 1.000 \\
C & $10 / 10(100.0 \%)$ & $10 / 10(100.0 \%)$ & $6 / 10(60.0 \%)$ & $\mathbf{0 . 0 2 3}$ \\
D & $10 / 10(100.0 \%)$ & $10 / 10(100.0 \%)$ & $3 / 10(30.0 \%)$ & $<\mathbf{0 . 0 0 1}$ \\
E & $10 / 10(100.0 \%)$ & $10 / 10(100.0 \%)$ & $3 / 10(30.0 \%)$ & $<\mathbf{0 . 0 0 1}$ \\
F & $10 / 10(100.0 \%)$ & $4 / 10(40.0 \%)$ & $0 / 10(00.0 \%)$ & $<\mathbf{0 . 0 0 1}$ \\
G & $10 / 10(100.0 \%)$ & $10 / 10(100.0 \%)$ & $1 / 10(10.0 \%)$ & $<\mathbf{0 . 0 0 1}$ \\
H & $10 / 10(100.0 \%)$ & $10 / 10(100.0 \%)$ & $5 / 10(50.0 \%)$ & $\mathbf{0 . 0 0 5}$ \\
All & $80 / 80(100.0 \%)$ & $74 / 80(92.5 \%)$ & $30 / 80(37.5 \%)$ & $<\mathbf{0 . 0 0 1}$ \\
\hline Statisti
\end{tabular}

Statistically significant $p$-values are bold.

†Fisher's exact test. was taken capturing the largest number of SO droplets per solution drop (ten drops onto each glass slide) for each syringe. The number of droplets of SO, when present, was analysed by the investigator who prepared the syringes (CSDJr) and another blinded examiner (GBM).

\section{Fourier-transform infrared spectroscopy (FTIR)}

A sample of each of the eight syringe models was studied by FTIR. After the plunger was removed, the rubber tip was gently rubbed onto a cesium iodide crystal to transfer the possible content of SO present in the syringe. Identification was performed using the Nicolet iS10 FTIR (Thermo Electron Scientific Instruments LLC, Madison, Wisconsin, USA) with bands ranging from $4000 / \mathrm{cm}^{-1}$ to $400 / \mathrm{cm}^{-1}$, resolution of $4 / \mathrm{cm}^{-1}$ and 32 scans.

\section{Statistical analysis}

Data analysis was performed qualitatively and quantitatively using the STATA 14.0 software (StataCorp LP, College Station, TX, USA). The presence/absence of SO droplets was analysed in terms of percentage in each type of syringe (A, B, C, D, E, F, G, and H) and under different conditions (positive control and with or without flicking). The comparison between the different levels of the variables of interest was performed using the Fisher's exact test. Firth's logistic regression was used to evaluate the presence/absence of SO droplets (dependent variable) in relation to the syringe types and evaluation conditions (predictive variables), presented as odds ratio. The number of oil droplets in each syringe was evaluated and compared between different syringe types and evaluation conditions from the Kruskal-Wallis test. The Dunn test was used as a post hoc analysis. For all the tests, a significant $P$ value was considered when lower or equal to 0.05 .

\section{Results}

A total of 240 syringes were analysed: 30 of each 8 models (A, B, C, D, E, F, G, and H). Ten syringes were evaluated in three different conditions (positive control, with agitation, and without agitation).

\section{Qualitative analysis}

The presence of SO droplets in each type of syringe and condition is shown in Table 2.

The presence of SO droplets was observed in all positive controls. When agitated by flicking, $100 \%$ of the samples of the syringes disclosed SO, except for type F (BD Plastipak), which presented $40 \%$ of positivity. Without agitation, a smaller percentage of samples with SO were observed, except for type B (Solidor), which presented $100 \%$ of positivity. The Fisher's exact test showed a significant difference for all syringe models, except for type B (100\% of samples with $\mathrm{SO}$ ).

Firth's logistic regression for the presence of oil droplets as response variable and syringe model and condition as predictive variables is presented in Table 3 . The F syringe was used as the reference because it presented a lower percentage of oil.

According to the syringe model, it was observed that all of them had a greater chance of presenting SO droplets when compared with type $\mathrm{F}(p<0.05)$, when the condition was controlled. Regarding the condition, the logistic regression adjusted for the syringe type indicated that the syringes subjected to agitation by flicking had a 265 -fold greater chance of presenting oil droplets when compared with the syringes without flicking $(\mathrm{OR}=263.18 ; 95 \% \mathrm{CI}$ : 15.43-4490.00; $P<0.001)$. As expected, the positive controls had a much higher likelihood of presenting oil droplets when compared with the syringe without flicking (OR = 7838.06; 95\% CI: 125.42-489835.90; $P<0.001)$. 


\section{Quantitative analysis}

Quantitative data on the number of SO droplets in each syringe (A, B, C, D, E, F, G, and H) under different conditions (positive control, agitation, and without agitation) are shown in Table 4. The Kruskal-Wallis' test was used to evaluate the differences between the number of SO droplets between the types of syringe and the handling conditions.

Statistically significant differences were observed between at least two conditions $(p<0.05)$ that were assessed by the Dunn's test for all types of syringes. For syringes $\mathrm{A}, \mathrm{B}, \mathrm{C}, \mathrm{D}, \mathrm{F}, \mathrm{G}$, and $\mathrm{H}$, there was a significant difference between the three conditions $(P<0.05)$. Syringe $\mathrm{E}$ presented a smaller number of droplets in the no-agitation condition when compared with the control $(P<0.001)$ and to the agitation one $(P<0.001)$. Syringe $\mathrm{F}$ showed a greater number of droplets in the positive controls in comparison with both agitation $(p<0.001)$ and no-agitation $(P<0.001)$.

Table 3 Firth's logistic regression for presence of silicone oil droplets adjusted for syringe type and condition.

Odds ratio $\begin{aligned} & 95 \% \text { confidence } \quad P \text { value } \\ & \text { interval }\end{aligned}$

\begin{tabular}{llllr}
\hline Syringes & & & & \\
A & 109.81 & 4.16 & 2896.55 & 0.005 \\
B & 7913.15 & 121.85 & 513872.60 & $<0.001$ \\
C & 544.02 & 20.51 & 14432.62 & $<0.001$ \\
D & 175.15 & 6.68 & 4590.66 & 0.002 \\
E & 175.15 & 6.68 & 4590.66 & 0.002 \\
F & Ref & - & - & - \\
G & 58.44 & 2.36 & 1449.81 & 0.013 \\
H & 376.46 & 14.38 & 9853.04 & $<0.001$ \\
Condition & & & & \\
Positive control & 7838.06 & 125.42 & 489835.90 & $<0.001$ \\
Agitation & 263.18 & 15.43 & 4490.00 & $<0.001$ \\
Without agitation & Ref & - & - & - \\
\hline
\end{tabular}

However, there was no statistically significant difference between agitation and no-agitation $(P=0.135)$.

\section{Fourier-transform infrared spectroscopy (FTIR)}

Analysis of the plunger tip by FTIR indicated the presence of polysiloxane (SO) in all models of syringe (Fig. 1). Although polysiloxane was the predominant compound, other compounds were found in small proportions: ester carbonyl (syringes A and F) and carboxylic acid salt carbonyl (syringes E, F, and G).

\section{Discussion}

Previous publications have reported the presence of SO droplets in the vitreous of individuals previously treated with intravitreal injections, assuming that oil comes from the interior of the syringes used in this procedure [4-7]. The present study identified the presence and release of SO in various models of syringe used for intravitreal injections under different handling conditions, especially after agitation by flicking.

In the positive controls, all ten syringe samples of each brand released SO droplets. When they were subjected to agitation by flicking in the study group, all syringes, except for six samples of the BD Plastipak, released droplets of SO. Without agitation, the syringes that released the smallest number of SO droplets were, respectively, the BD Plastipak (F), SR (G), and Descarpack (A). Notably, we did not verify whether these differences were consistent across multiple lots. Our previous studies have also shown similar results $[9,10]$.

The choice of syringes for this study was based on the models most commonly used in some European countries, North America, and Brazil. BD Ultra-Fine, although manufactured in the USA, is widely found and used in Brazil, and also is frequently sold with prefilled bevacizumab by
Table 4 Quantitative analysis of the number of silicone oil droplets in each syringe under different handling conditions.

\begin{tabular}{lclll}
\hline $\begin{array}{l}\text { Syringe/ } \\
\text { condition }\end{array}$ & $\begin{array}{l}\text { Positive control mean } \pm \\
\text { SD (median) }\end{array}$ & $\begin{array}{l}\text { Agitation mean } \pm \text { SD } \\
\text { (median) }\end{array}$ & $\begin{array}{l}\text { Without agitation mean } \pm \\
\text { SD (median) }\end{array}$ & $P$ value $^{\dagger}$ \\
\hline A & $53.00 \pm 24.54(46.50)$ & $6.60 \pm 3.53(6.00)$ & $0.20 \pm 0.42(0.00)$ & $<\mathbf{0 . 0 0 0 1}$ \\
B & $79.20 \pm 19.62(77.00)$ & $9.10 \pm 3.14(9.00)$ & $2.70 \pm 1.70(2.50)$ & $<\mathbf{0 . 0 0 0 1}$ \\
C & $131.90 \pm 40.61(119.50)$ & $68.60 \pm 21.52(68.50)$ & $1.20 \pm 1.32(1.00)$ & $<\mathbf{0 . 0 0 0 1}$ \\
D & $2.20 \pm 0.79(2.00)$ & $15.80 \pm 4.87(15.00)$ & $0.30 \pm 0.48(0.00)$ & $<\mathbf{0 . 0 0 0 1}$ \\
E & $32.90 \pm 7.52(32.50)$ & $26.00 \pm 11.70(22.00)$ & $0.30 \pm 0.48(0.00)$ & $<\mathbf{0 . 0 0 0 1}$ \\
F & $17.90 \pm 9.29(18.50)$ & $0.60 \pm 0.84(0.00)$ & $0.00 \pm 0.00(0.00)$ & $<\mathbf{0 . 0 0 0 1}$ \\
G & $80.70 \pm 14.67(75.50)$ & $15.00 \pm 36.67(14.50)$ & $0.10 \pm 0.32(0.00)$ & $<\mathbf{0 . 0 0 0 1}$ \\
H & $59.60 \pm 8.92(57.00)$ & $57.20 \pm 14.50(54.50)$ & $0.60 \pm 0.70(0.50)$ & $<\mathbf{0 . 0 0 0 1}$ \\
$\boldsymbol{P}$ value $^{\dagger}$ & $<\mathbf{0 . 0 0 0 1}$ & $<\mathbf{0 . 0 0 0 1}$ & $<\mathbf{0 . 0 0 0 1}$ & \\
\hline
\end{tabular}

${ }^{\dagger}$ Kruskal-Wallis test. 

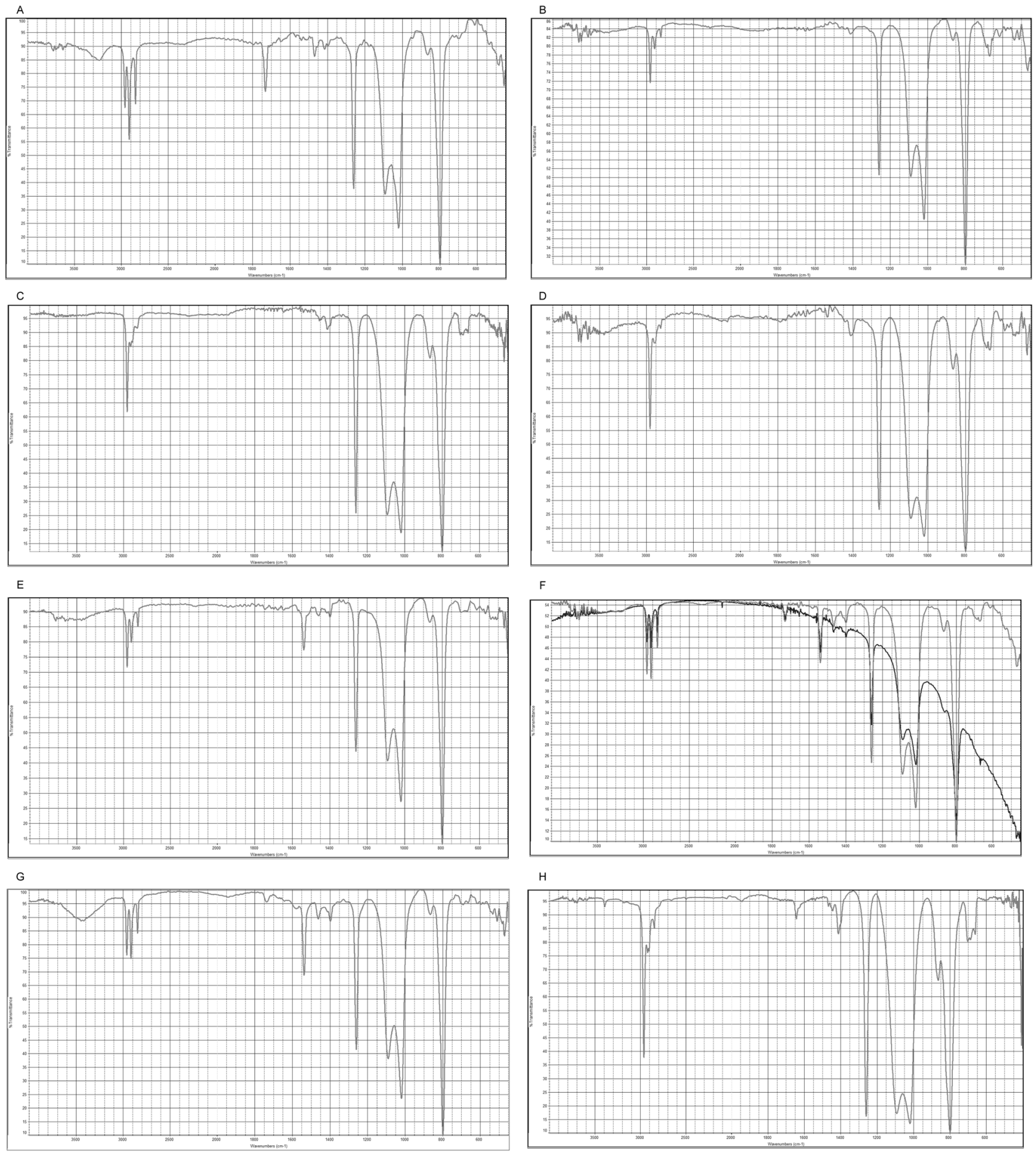

Fig. 1 Fourier-transform infrared spectroscopy graph shows the characteristic bands of polysiloxane (silicone oil) in all syringes (A, B, C, D, E, F, G, H).

compounding pharmacies in the USA. BD Plastipak is widely available in Europe, in Canada, and in Brazil. The others are more easily found in Brazil.

Emerson (2017) reported that some BD insulin syringes also released SO droplets [11]. The experiments were performed under steady-state conditions of manipulation of the syringes. It was speculated that the absence of a dead space in that specific syringe model was the cause. Theoretically, the dead space serves as a trap for high-resistance components, such as SO droplets.

Other studies have shown that insulin syringes release more SO droplets when subjected to more vigorous agitation, especially after agitation by flicking, compared with the syringes without prior agitation $[9,12,13]$. The 
practice of flicking the syringe during preparation of the medication is quite common, as an attempt to remove air bubbles from the syringe prior to injection. In addition, priming the plunger, different temperatures, and the presence of air bubbles (without agitation) had little impact on the release of SO by the syringes [12]. In the agitation groups, the presence of air also showed an incremental effect on the release rate [12]. Another study from our group also demonstrated the presence of SO obtained from the outer surface of 26- and 30-gauge needles commonly used to be attached to the syringes for injection [10]. A recent study from our group has shown no significant amount of SO released by the inner surface of most commercially available needles (unpublished data). This finding, added to the fact that the total area of the needle inner surface is much smaller than that of any syringe, makes us believe that the needles did not have any significant impact on the main findings of the study.

Other studies unrelated to ophthalmology [14-16] corroborate the fact that there is a greater release of SO from the inner walls of the syringes when they are agitated. In addition, the formation of protein aggregates is described from the interaction of certain medications with $\mathrm{SO}$, air bubbles, and agitation. A similar process can occur with intravitreal injections, causing serious and previously unanticipated damage to eye.

FTIR confirmed the presence of polysiloxane (SO) at the tip of the plunger of all models plus two other compounds in smaller amounts: ester carbonyl (A and F) and carboxylic acid salt carbonyl (E, F, and G). Neither do they come from the production of silicone nor represent possible impurities. Further studies are required to understand it.

With the increasing number of intravitreal injections, the number of individuals with SO droplets in the vitreous and relevant complaint also increases. Further complications may occur when vitrectomy is required to treat floaters secondary to SO droplets in the vitreous. In addition, retinal specialists worldwide are concerned about the occurrence of ocular inflammation without clearly defined etiology after intravitreal injections of antiangiogenic drugs. Noninfectious vitritis was reported in $0.10 \%$ of cases after 66,356 injections of bevacizumab, $0.02 \%$ after 26 injections of ranibizumab (Lucentis, Genentech), and $0.16 \%$ after 8,071 injections of aflibercept (Eylea, Regeneron Pharmaceuticals, Tarrytown, NY) [17]. The American Society of Retinal Specialists Therapeutic Surveillance Committee also reported cases of sterile inflammation related to aflibercept without a clear explanation suggested by the authors [18]. Our group also identified six cases of ocular inflammation after aflibercept injections with the use of the SR syringe [3], which was later proven to release SO $[9,10,12]$. Since then, our group recommends that the syringes not be agitated during its handling for intravitreal injection, as it is speculated that the release of SO droplets may contribute to the inflammatory reaction. In agreement with this hypothesis, some studies have reported a more intense protein aggregation and the formation of insoluble molecules resulting from the agitation of the syringes in the presence of SO from the syringes [14-16].

Finally, it has also been reported that SO droplets can act as immunological adjuvants in dermatological procedures performed in the subcutaneous space in mice $[19,20]$. In addition, a recently published study showed undesirable effects of SO on therapeutic proteins, including adsorption to the oil droplets and increased secretion of several innate cytokines from human peripheral blood mononuclear cells [21]. The authors concluded that the SO droplets form complexes with pharmaceutical proteins that can potentially invoke early- and late-stage immune responses [21].

The BD Plastipak syringe was shown to release some SO after agitation, which differs from one previous study of our group [9]. Not only was the size sample of the current study larger but also examiners were more trained than in the initial research period. In addition, FTIR showed confirmed that the BD Plastipak syringe really has SO on the tip of its plunger [13].

A limitation of this study was the quantitative analysis of the SO droplets, since the variation in size of these droplets was not taken into consideration and only the field with the highest number of SO droplets was chosen for quantification. A more accurate quantitative analysis test would be needed to draw more definite conclusions about the amount of SO. However, it does not invalidate the current findings because the qualitative analysis of the syringe samples with the presence of SO was reliable and reproducible.

In conclusion, syringes commonly used for intravitreal injections frequently release SO droplets, especially when agitated. In order to prevent potential hazards to the eye, we recommend that the syringes not be agitated at the time of the injection.

\section{Summary}

\section{What was known before}

- SO might be seen in the vitreous-Syringes seem to be the source

\section{What this study adds}

- Most syringes have and release SO-Agitation of the syringes causes additional release of $\mathrm{SO}$ 
Author contributions All authors conceived and/or designed the work that led to the submission, acquired data, and/or played an important role in interpreting the results, drafted or revised the paper, approved the final version, and agreed to be accountable for all aspects of the work.

\section{Compliance with ethical standards}

Conflict of interest The authors declare that they have no conflict of interest.

Publisher's note Springer Nature remains neutral with regard to jurisdictional claims in published maps and institutional affiliations.

\section{References}

1. Grzybowski A, Told R, Sacu S, Bandello F, Moisseiev E, Loewenstein A, et al. 2018 Update on Intravitreal Injections: euretina expert consensus recommendations. Ophthalmologica. 2018;239:181-93.

2. Tah V, Orlans HO, Hyer J, Casswell E, Din N, Sri Shanmuganathan $\mathrm{V}$, et al. Anti-VEGF therapy and the retina: an update. $\mathrm{J}$ Ophthalmol. 2015;2015:627674.

3. Melo GB, Figueira ACM, Batista FAH, Lima Filho AAS, Rodrigues EB, Belfort $\mathrm{R} \mathrm{Jr}$, et al. Inflammatory reaction after aflibercept intravitreal injections associated with silicone oil droplets released from syringes: a case-control study. Ophthalmic Surg Lasers Imaging Retin. 2019;50:288-94.

4. Khurana RN, Chang LK, Porco TC. Incidence of presumed silicone oil droplets in the vitreous cavity after intravitreal bevacizumab injection with insulin syringes. JAMA Ophthalmol. 2017;135:800-3.

5. Bakri SJ, Ekdawi NS. Intravitreal silicone oil droplets after intravitreal drug injections. Retina. 2008;28:996-1001.

6. Avery RL, Castellarin AA, Dhoot DS, Pieramici DJ, Nasir MA, Steinle NC et al. Large silicone droplets after intravitreal bevacizumab (Avastin). Retin Cases Brief Rep. 2019;13:130-4. https://doi.org/10.1097/ICB.0000000000000570.

7. Yu JH, Gallemore E, Kim JK, Patel R, Calderon J, Gallemore RP. Silicone oil droplets following intravitreal bevacizumab injections. Am J Ophthalmol Case Rep. 2017;10:142-4.

8. Stone TW, editor. ASRS 2018 Preferences and trends membership survey. Chicago, IL: American Society of Retina Specialists;
2018. https://www.asrs.org/content/documents/_2018-pat-surveyresults-for-website.pdf. Accessed 29 Aug 2018.

9. Melo GB, Dias CS Jr, Carvalho MR, Cardoso AL, Morais FB, Figueira ACM, et al. Release of silicone oil from syringes. Int $\mathbf{J}$ Retin Vitreous. 2019;5:1.

10. Melo GB, Emerson GG, Lima Filho AAS, Ota S, Maia M. Needles as a source of silicone oil during intravitreal injection. Eye. 2019;33:1025-7.

11. Emerson GG. Silicone oil droplets are more common in fluid from BD insulin syringes as compared to other syringes. J VitreoRet Dis. 2017;1:401-6.

12. Melo GB, Emerson GG, Dias CS Jr, Morais FB, Lima Filho A, de $\mathrm{S}$, et al. Release of silicone oil and the off-label use of syringes in ophthalmology. Br J Ophthalmol. 2019. https://doi.org/10.1136/ bjophthalmol-2019-313823.

13. Agra LLM, Melo GB, Lima Filho AAS, Ota S, Maia M. Silicone oil found in syringes commonly used for intravitreal injections. Arq Bras Oftalmol. 2019;82:354-5.

14. Krayukhina E, Tsumoto K, Uchiyama S, Fukui K. Effects of syringe material and silicone oil lubrication on the stability of pharmaceutical proteins. J Pharm Sci. 2015;104:527-35.

15. Gerhardt A, Mcgraw NR, Schwartz DK, Bee JS, Carpenter JF, Randolph TW. Protein aggregation and particle formation in prefilled glass syringes. J Pharm Sci. 2014;103:1601-12.

16. Teska BM, Brake JM, Tronto GS, Carpenter JF. Aggregation and particle formation of therapeutic proteins in contact with a novel fluoropolymer surface versus siliconized surfaces: effects of agitation in vials and in prefilled syringes. J Pharm Sci. 2016;105:2053-65.

17. Williams PD, Chong D, Fuller T, Callanan D. Noninfectious vitritis after intravitreal injection of anti-VEGF agents. Variations in rates and presentation by medication. Retina. 2016;36:909-13.

18. Hahn P, Chung MM, Flynn HW Jr., Huang SS, Kim JE, Mahmoud $\mathrm{TH}$, et al. Postmarketing analysis of aflibercept-related sterile intraocular inflammation. JAMA Ophthalmol. 2015;133:421-6.

19. Chisholm CF, Nguyen BH, Soucie KR, Torres RM, Carpenter JF, Randolph TW. In vivo analysis of the potency of silicone oil microdroplets as immunological adjuvants in protein formulations. J Pharm Sci. 2015;104:3681-90.

20. Wambier CG, Danilau Ostroski TK, Perillo de Farias Wambier S, Beltrame FL, Cappel MA, Piñeiro Maceira JM. Syringe lubricant and adverse reactions. Int J Dermatol. 2018;57:122-3.

21. Krayukhina E, Yokoyama M, Hayashihara KK, Maruno T, Noda $\mathrm{M}$, Watanabe $\mathrm{H}$, et al. An assessment of the ability of submicronand micron-size silicone oil droplets in dropped prefillable syringes to invoke early- and late-stage immune responses. J Pharm Sci. 2019;108:2278-87. 